\title{
Toxicovigilance: Experience of the Tygerberg Poison Information Centre in context
}

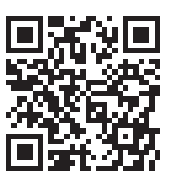

Poison information centres are confronted daily by reports of, and requests for, support after alleged exposures to various types of toxins. These emanate from healthcare professionals, veterinarians, members of the public, farmers, scientists and activists, most of whom believe their own diagnosis to be correct. Based on their diagnoses, often erroneous, such individuals often prescribe or administer a range of treatments, many of which are incorrect. Furthermore, people who may not have been exposed to a clinically significant dose of toxins, or in fact any toxins at all, often unjustifiably fear for their health or lives as a result of alarmist statements by activists.

Clinical staff and scientists who field calls to poison information centres (PICs) have the challenge of maintaining an objective approach to every single report of excessive human exposure to a toxin. False information, common beliefs held by the public, poor diagnosis and treatment, media outcries about poisoning, and lack of knowledge and understanding of toxins and poisoning are reflected in a high proportion of poisoning incidents that are reported to PICs. False or incorrect information about pesticides is fuelled by activists who seek to have agricultural remedies or pesticides removed from the market, or their general use at least minimised.

The research conducted by the Tygerberg Poison Information Centre (TPIC) and reported in this issue of $S A M J^{1,2]}$ is extremely valuable. It not only places indisputable facts on the table with regard to human poisoning, but also identifies issues that need to be addressed concerning pesticides, stock remedies (veterinary medicines), petroleum fuels, industrial chemicals and household chemicals.

The first and perhaps most valuable contribution in these articles is the classification of human poisoning incidents. Most healthcare professionals and members of the public are under the wrongful impression that pesticides are responsible for most human poisoning incidents and that the annual number of incidents is vast. A further erroneous belief is that normal workplace application of pesticides is responsible for the poisoning of a large number of farm workers and farmers, other workers and urban residents who live close to farms.

Results of the TPIC studies show beyond any doubt that pesticides are not the main agents in human poisoning. Rather, pesticide exposure is the result of off-label use of pesticides (use for purposes, and in ways, other than those prescribed according to their labels). The experience of the Griffon Poison Information Centre (GPIC) corroborates this particular finding, with fewer than $1 \%$ of pesticide exposures resulting from labeldirected use. The Act that regulates pesticides in South Africa, the Fertilisers, Farm Feeds, Agricultural Remedies and Stock Remedies Act, 1947 (Act No. 36 of 1947), forbids the use of any pesticide or veterinary medicine for purposes or in ways other than those directed on the labels. Off-label use in suicide attempts, incorrect treatment of household and garden pests, unsafe storage of pesticides, illegal sales of decanted pesticides in unmarked containers, homicides, excessive use of pesticides in home and home garden applications, illegal storage in unmarked food and beverage containers, and blatant ignorance are all examples of ways in which unregulated human exposure to pesticides occur, leading to anything from mild allergic responses to life-threatening poisoning.

It is understandable that healthcare professionals may have insufficient knowledge about pesticides to be able to arrive at the correct diagnosis of toxicity in every patient. Nor are they necessarily able to classify the agent(s) responsible for the patient's illness accurately. Failure to seek expert advice from a PIC can result in incorrect treatment that may even worsen the patient's condition. The scarcity of fully operational PICs left in South Africa, the Department of Health's reluctance to support such centres, and its failure to maintain a database of human poisoning incidents add to the challenges in managing human poisoning incidents effectively. All of these challenges lead to incorrect information being filtered back to the general public, with resultant unjustified attacks on the pesticide and agricultural sector. What is also alarming is that rural healthcare facilities often have little or nothing in the way of antidotes, leaving healthcare professionals in a serious predicament when a patient needs urgent treatment.

What the TPIC studies also show is the compounding effect of prescription drugs, narcotics, alcohol abuse and undue exposure to other chemicals. It is troubling that one of the most widely prescribed and used analgesics, namely acetaminophen (paracetamol), is implicated in a vast number of cases of human poisoning. This valuable pain killer is branded by manufacturers as a safe medicine despite this large annual occurrence of poisoning. In the experience of the GPIC, which deals mostly with cases of pesticide poisoning, paracetamol, anti-inflammatory drugs and antifertility drugs are regularly reported in unprescribed intake. Paraffin is another chemical with a high incidence of ingestion by humans.

These findings point to one common factor: end-users of pesticides, corrosive and abrasive chemicals, petroleum fuels, industrial chemicals and veterinary medicines are highly irresponsible in the way in which they store and use these products. Safe storage does not exist in most households, leading to risky exposure of children and domestic animals to these products. Pesticides, petroleum fuels, prescription medications, narcotics, household chemicals and veterinary medicines are seldom stored safely, as directed on their labels, leading to poisoning incidents. Sale of many of these commodities by street vendors, without advice on their safe storage and use, adds to the problem.

It is interesting to compare South Africa with Brazil and Iran. In both the latter countries, large numbers of pesticide poisoning cases are reported, most involving workers who are exposed to pesticides unnecessarily owing to incorrect use. One would expect South Africa, as a developing country, to report the same experience, but this is thankfully not the case. The TPIC studies, and the GPIC experience, are in agreement that the majority of pesticide poisoning incidents occur in the home and home garden sector. Agriculture contributes virtually nothing to pesticide poisoning in our country.

As far as improving the situation is concerned, it is futile to expect state departments to put measures into effect. The compulsory reporting of human poisoning incidents to the Department of Health (DoH) by healthcare professionals has failed. The Department of Environmental Affairs (DoEA), as competent authority for chemical safety, has no programme in place to promote chemical safety. The inspectorates of both the DoH and the Department of Agriculture (DoA) are so severely understaffed that they can have little impact on illegal sales, illegal use, illegal possession and blatant misuse of human medicines, pesticides and veterinary medicines. The DoA does, however, alleviate the situation by ensuring that the use of certain products that are blatantly misused is severely restricted. An example is chlorpyrifos, an organophosphate insecticide that was implicated in more than half of human and pet poisoning cases in the urban arena. Within 3 months after its sale and use in the home and home garden sector was formally prohibited, the incidence rate dropped to less than $5 \%$ of cases.

It may be valuable to list those toxic agents that compete for the largest portion of poisoning incidents and work towards restrictive 
sales of such products, meaning that they should only be sold with specific instructions for their safe storage and responsible use.

More emphasis should be placed on knowledge of basic toxicology in the training of all healthcare professionals and veterinarians, so that they are capable of effective diagnosis and treatment of poisoning.

Lastly, I would like to commend the TPIC on their studies, as the record is set straight in terms of human poisoning. It is not the products that are to be blamed, but rather people with insufficient knowledge who are allowed access to them and then use them without taking note of accompanying label or prescription instructions.

\section{G H Verdoorn}

Director: Griffon Poison Information Centre, Randburg, Gauteng, South Africa

\section{Corresponding author: G H Verdoorn (nesher@tiscali.co.za)}

1. Veale DJH, Wium CA Müller GJ. Toxicovigilance I: A survey of acute poisonings in South Africa based on Tygerberg Poison Information Centre data. S Afr Med J 2013;103(5):293-297. [http://dx.doi. org/10.7196/SAMJ.6647]

2. Veale DJH, Wium CA, Müller GJ. Toxicovigilance II: A survey of the spectrum of acute poisoning and current practices in the initial management of poisoning cases admitted to South African hospitals. SAfr Med J 2013;103(5):298-303. [http://dx.doi.org/10.7196/SAMJ.6648]

S Afr Med J 2013;103(5):288-289. DOI:10.7196/SAMJ.6840 